\title{
Erratum to: Fingerprint liveness detection using gradient-based texture features
}

\author{
Zhihua Xia ${ }^{1}$ - Rui Lv ${ }^{1}$ - Yafeng $Z_{h u^{1}}$. \\ Peng $\mathrm{Ji}^{1}$ - Huiyu Sun ${ }^{2}$ - Yun-Qing Shi ${ }^{3}$
}

Published online: 25 August 2016

(C) Springer-Verlag London 2016

\section{Erratum to: SIViP \\ DOI 10.1007/s11760-016-0936-z}

In the original publication, the reference Gragnaniello et al. cited in the "Related works" section has been missed in the references list. The correct reference is given below.

\section{Reference}

1. Gragnaniello, D., Poggi, G., Sansone, C., Verdoliva, L.: Local contrast phase descriptor for fingerprint liveness detection. Pattern Recognit. 48(4), 1050-1058 (2015)

The online version of the original article can be found under doi:10.1007/s11760-016-0936-z.

Zhihua Xia

xia_zhihua@163.com

1 Jiangsu Engineering Center of Network Monitoring, Jiangsu Collaborative Innovation Center on Atmospheric

Environment and Equipment Technology, College of

Computer and Software, Nanjing University of Information

Science and Technology, Nanjing 210044, China

2 Department of Computer Science, New York University, New York, NY 10012, USA

3 Department of Electrical and Computer Engineering, New Jersey Institute of Technology, New York, NJ 07102, USA 